Research Article

\title{
Network Pharmacology and Molecular Docking Study of Zhishi-Baizhu Herb Pair in the Treatment of Gastric Cancer
}

\author{
Ying Qu $\mathbb{D}^{1},{ }^{1}$ Xiangyang Yang, ${ }^{1}$ Jingxiang Li, ${ }^{1}$ Shuxin Zhang ${ }^{\mathbb{D}},{ }^{1}$ Shiying Li, \\ Mengyuan Wang, ${ }^{1}$ Lu Zhou $\mathbb{D}^{2}{ }^{2}$ Zhiying Wang, ${ }^{1}$ Zi Lin, ${ }^{1}$ Yuzhang Yin, ${ }^{1}$ Jinlong Liu, \\ Nan Wang, ${ }^{1}$ and Yang Yang \\ ${ }^{1}$ Dongzhimen Hospital, Beijing University of Chinese Medicine, Beijing 100700, China \\ ${ }^{2}$ School of Chinese Medicine, Beijing University of Chinese Medicine, Beijing 100029, China \\ Correspondence should be addressed to Shuxin Zhang; zhshxincn@126.com
}

Received 11 September 2021; Revised 20 October 2021; Accepted 2 November 2021; Published 2 December 2021

Academic Editor: K. Arunachalam

Copyright ( $\odot 2021$ Ying Qu et al. This is an open access article distributed under the Creative Commons Attribution License, which permits unrestricted use, distribution, and reproduction in any medium, provided the original work is properly cited.

\begin{abstract}
Objective. This study aimed to investigate the possible mechanism of the Zhishi and Baizhu herb pair in the treatment of gastric cancer by means of network pharmacology and molecular docking and to provide a theoretical basis for experiments and clinical application of traditional Chinese medicine for treating gastric cancer. Methods. The main active chemical components of Zhishi and Baizhu were screened through Traditional Chinese Medicine Systems Pharmacology (TCMSP) database and selected by using the thresholds of oral bioavailability $\geq 30 \%$ and drug-likeness $\geq 18 \%$. The targets of Zhishi and Baizhu were obtained from TCMSP, Therapeutic Targets Database (TTD), and the DrugBank database. The corresponding genes of the targets were retrieved from the UniProt database, and the gastric cancer targets were obtained from the GeneCards database and TTD. Subsequently, the networks were built between the main drug components, drug targets, and gastric cancer targets. Then, the enrichment analyses of GO and KEGG were applied to predict the potential roles of gastric cancer pathogenesis via the R package clusterProfiler. Finally, molecular docking was used to determine the affinity between the targets and components. Results. Twenty-seven main active components were predicted from the Zhishi-Baizhu herb pair, and a total of 120 intersection genes were screened from 303 potential medicine genes and 1,839 disease genes. The enrichment included the PI3K-Akt and IL-17 signaling pathways, and the network analysis showed that the Zhishi-Baizhu herb pair acted on seven key targets, namely, AKT1, MMP9, IL-6, CCND1, BCL2, MTOR, and MDM2 (where they played a role in treating gastric cancer). Molecular docking showed that luteolin and naringenin could stably bind to the targets. Conclusion. The possible mechanisms of the components of the Zhishi-Baizhu herb pair in treating gastric cancer might be related to luteolin and naringenin, which intervened with the targets AKT1, MMP9, IL-6, CCND1, BCL2, MTOR, and MDM2, and are linked with the PI3K-Akt and IL-17 signaling pathways. This knowledge will lay a solid foundation for further experimental and clinical studies.
\end{abstract}

\section{Introduction}

Gastric cancer (GC) is a common malignant tumour in the world today and ranks third in fatalities and fifth in prevalence among malignant tumours [1]; it is the focus of clinical, epidemiology, and translational research at present [2].

Currently, surgical resection, perioperative adjuvant or neoadjuvant radiotherapy, immunotherapy, molecular targeted agents, and chemotherapy are the most effective treatments for this disease [3-5]. However, the poor prognosis at present may be due to the multiple-drug resistance of GC cells. Traditional Chinese medicine has been used to treat GC for thousands of years and is guided by a holistic concept, not only for local treatment but also for regulating the state of the whole body to control the development of a tumour [6,7].

In recent years, the position of Chinese medicine in treating GC has also become increasingly crucial. As reported in previous studies, Chinese medicine can effectively 
inhibit the invasion and metastasis of GC cells and greatly improve the prognosis of GC patients, subsequently improving the quality of the lives of patients $[8,9]$.

In traditional Chinese medicine, GC belongs to the category of gastralgia, dysphagia, stomach reflux, abdominal mass, and others [10]. Its main pathogenesis is due to weakness of the spleen and stomach, phlegm and blood stasis, and deficiency-excess mixing. This leads to a disturbance of the circulation of qi, blood and body fluids, and an accumulation of water in the stomach. Additionally, toxins persist from stasis, which transform into cancer after a long time [11].

The herbal pairing of Zhishi (Aurantii Fructus Immaturus) and Baizhu (Atractylodes macrocephala Koidz.) has been frequently used for treating digestive diseases. This pairing was found by a famous Chinese physician named Zhang Zhonging in the Eastern Han Dynasty [12], and, with its ideal curative effect, some later physicians developed its use in the treatment of GC. It also has a satisfactory clinical effect $[13,14]$.

Zhishi has a slightly cold nature (Chinese medicine theory) and a bitter, pungent, and acidic flavour, with a meridian tropism in the stomach and spleen. It is used for treating abdominal mass, dissipating phlegm, resolving mass issues, and settling stomach-Qi $[15,16]$. As "Yao Pin Hua Yi" recorded, "Zhishi was especially used to remove the pathogenic factors of the stomach and to remove the agglomerate. The main therapeutic effect of Zhishi was in Zhongwan (epigastrium) to treat the blood level and to deal with the full pathogenic factors between umbilicus and stomach. It is also used to eliminate phlegm and remove the stagnation of pathogenic Qi in chest" [17]. Previous studies verified that flavonoids in Zhishi have anticancer effects and regulate cancer cell apoptosis and metastasis effectively by in vitro and in vivo experiments [18], and Yang Dong proved that sinensetin could inhibit the proliferation of human AGS GC cells and lead to cell cycle arrest in the G2/M phase and induce apoptosis [19].

Baizhu was first discovered in Shennong's Classic of Materia Medica as an upper grade drug; it has a warm nature and a bitter, sweet taste, with a meridian tropism in the stomach and spleen. It can invigorate the spleen and replenish $\mathrm{Qi}$, induce diuresis, stop perspiration, dry dampness of the body, and calm a foetus [20]. The detailed description related to Baizhu was "dispelling dehumidification, harmonizing spleen and replenishing qi, strengthening the spleen and stomach, and promoting the appetite" in "Yi Xue Qi Yuan," and "Changsha Yao Jie" said "Baizhu enters the Stomach Meridian of Foot-Yangming and the Spleen Meridian of FootTaiyin, with the use of dispelling dampness and causing dryness, invigorating spleen and replenishing qi, and so on. It can nourish stomach-Qi powerfully, reduce turbid Yin to promote the appetite, and it is good at preventing vomiting" [21]. Modern pharmacological studies have reported that Baizhu and its active sites have been shown to have anti-GC effects. Atractylenolide I can inhibit the growth and reproduction of GC MGC-803 cells, and atractylenolide II can induce apoptosis in the GC cell lines HGC-27 and AGS. Serum containing Baizhu can facilitate the apoptosis of GC cells line SGC-7901, side population cells, and nonside population cells. It can also inhibit the ability of cell proliferation and tumour formation. Furthermore, Baizhu can ameliorate the symptoms of patients with cachexia caused by GC and improve their appetite and body function state [22].

The compatibility of Zhishi and Baizhu is a classical prescription and the pairing had long been used to cure internal-injured spleen-stomach diseases [23], and the composition of these two drugs originated from the Zhi-Zhu decoction in the Synopsis of Golden Chamber. The Zhi-Zhu decoction consisted of seven Zhishi and two taels of Baizhu, which was mainly used to cure lumps under the heart. Many experiences have been summarised through the research of later generations of the physicians who used the derivate prescriptions of Zhi-Zhu decoction to prevent and treat GC in experiments or clinical studies and have achieved good therapeutic effects $[24,25]$. The mechanism of these two herbs for treating GC is not clear at present. Hence, the aim of our research is to further investigate the detailed mechanism of the active components of Zhishi and Baizhu to treat GC systematically by using network pharmacology, thereby providing new theoretical basis for traditional Chinese medicine in the treatment of GC.

Network pharmacology and molecular docking are new technologies based on systems biology and database molecular correlation analysis for the exploration of new drugs and prediction of their related targets [26]. Network pharmacology has been used to explore the active components and potential targets relevant to the treatment of GC with Zhishi and Baizhu. Molecular docking was performed to verify the detailed interactions, mainly for affinities between Zhishi and Baizhu and the candidate targets. This research not only provides a comprehensive understanding of the molecular mechanism of Zhishi and Baizhu acting on GC but also offers a rapid and effective strategy for further experimental and clinical research in the future [27].

\section{Materials and Methods}

2.1. Main Component Screening. The main chemical compounds of the Zhishi (ZS) and Baizhu (BZ) herb pair were acquired from the Traditional Chinese Medicine Systems Pharmacology (TCMSP, https://tcmspw.com/tcmsp.php) database [28], and the main active chemical components were screened based on the threshold values of oral bioavailability $(\mathrm{OB}) \geq 30 \%$ and drug-likeness (DL) $\geq 0.18$, and the targets of the components were obtained from the TCMSP database and the Therapeutic Targets Database (TTD, https://bidd.nus.edu.sg/group/ttd/ttd.asp) [29] and combined with DrugBank (https://www.drugbank.ca) [30]. Subsequently, we input each of the candidate targets into the UniProt database (https://www.uniprot.org/) [31] to screen the human genes related to those targets (the species option was "Homo sapiens"), and the GC-related human genes were downloaded from the GeneCards database (https://www. genecards.org/) [32] and TTD using the keyword "GC." Next, the candidate targets of the ZS-BZ herb pair against GC were obtained by taking an intersection of the above targets with a Venn diagram tool (https://bioinfogp.cnb.csic. es/tools/venny/index.html) [33]. 
2.2. Network Construction and Hub Gene Selection. We inputted these overlapped targets into STRING tools (https://string-db.org) [34] to construct the protein-protein interactions (PPI) network, and the required score was defaulted to be greater than 0.4. We selected "Homo sapiens" from species, and the compound-target-disease network was built and displayed using Cytoscape 3.6.1 software (https:// cytoscape.org/) [35]. We used the cytoHubba plugin to select the key genes, and a degree greater than 2-fold above the median was used to evaluate and sequence the central genes. We chose the top 20 main related genes [36].

2.3. Gene Ontology and Kyoto Encyclopedia of Genes and Genomes Pathways Enrichment Analysis. Gene ontology (GO) and Kyoto Encyclopedia of Genes and Genomes (KEGG) enrichment analyses of potential targets were performed using the $\mathrm{R}$ package.

ClusterProfiler was used to search the related functions and pathways [37]; we set the following values: width $=35$, height $=20$, units $=$ "cm," and $P$ value $<0.05$ as the condition to visualise the results. Three selected analysis items were selected for GO enrichment analysis: molecular functions, cell components, and biological processes. Thus, we obtained the important terms of enriched GO terms and KEGG pathways to study the specific mechanism of the ZS-BZ herb pair against GC.

2.4. Molecular Docking Technology. The 3D structures of the target proteins were downloaded from the RCSB PDB database (https://www.rcsb.org/) [38]; the source of organism was set as "Homo sapiens" and refined at resolutions of 2.0-3.0 $\AA$ [26], and the active compounds were downloaded from TCMSP and were used as ligands. Docking results were analysed using PyMOL 2.3.0 to reveal the 3D protein-ligand complex, and we used AutoDockTools 1.5.6 software to remove water molecules $[39,40]$, add nonpolar hydrogen, and then calculate the affinities between these proteins and ligands. We carried out hydrogenation and charge calculation of these 20 main target proteins. The docking binding energy based on the threshold conditions of $\leq-5.0 \mathrm{kcal} / \mathrm{mol}$ was considered successfully to predict well compoundprotein binding affinity [41], and the related conformations with good affinities were visualised in PyMOL 2.3.0.

\section{Results}

3.1. Targets of ZS-BZ Herb Pair against GC. Excluding no known targets of compounds, a total of 27 main chemical compounds from the ZS (20) and BZ (7) herb pair were obtained from the TCMSP database, and 743 targets were retrieved from the TCMSP database, TTD, and DrugBank using the names of all the selected compounds above. The main compounds were luteolin, naringenin, 12-senecioyl-2E,8E,10Eatractylentriol, nobiletin, and tetramethoxyluteolin. The selected compounds are shown in Table 1. Then, we put these targets into the UniProt database and selected species option as "Homo sapiens"; a total of 303 human genes related to these medicine targets were screened out. In addition, a total of 1,839 GCrelated targets were screened from the GeneCards database. All of the suitable herb targets are also shown in Table 1, and the predictive candidate targets of $\mathrm{ZS}$ and $\mathrm{BZ}$ overlapped with the GC-related targets. In total, 120 selected targets of ZS and BZ against GC were identified (excluding duplicate targets) and are shown in Figure 1.

\subsection{Results of Network}

3.2.1. Results of PPI Network. The STRING tool was applied to construct the PPI network of the herb compound-diseasetarget relationships for the 120 overlapping targets. We selected a score $>0.7$ from the analysis. The network of PPI contained 120 nodes and 671 edges, the average node of degree was 11.2, and the average local clustering coefficient was 0.533 (as shown in Figure 2).

3.2.2. Cytoscape Pharmacological Network Diagram of Targets and the Result of Hub Gene Selection. We used Cytoscape 3.6.1 software to visualise the score targets of the results of the STRING database of the ZS-BZ herb pair and gastric cancer (Figure 3). Then we set the style of the node colour and size to construct drug-target interactions of the drug and disease. It contained 123 nodes and 178 edges, the average node degree was 11.2, and the top 20 important targets with an average degree value greater than 2 times of 11.2 were selected by the cytoHubba plugin. These top 20 targets were the key targets of the ZS-BZ herb pair against gastric cancer; the darker the colour, the higher the correlation (Figure 4 and Table 2).

\subsection{Results of Enrichment Analysis}

3.3.1. GO Analysis. The multiple mechanisms of the ZS-BZ herb pair against gastric cancer were identified using the $\mathrm{R}$ package clusterProfiler of Programming Language, and 46 enriched GO terms were identified $(P<0.05)$. The results of GO analysis were separated into three parts, as follows: (1) biological processes: response to lipopolysaccharide, cellular response to drugs, regulation of apoptotic signaling pathway, response to nutrient levels, and regulation of DNA-binding transcription factor activity (Figure 5(a)); (2) cellular components: membrane rafts, cyclin-dependent protein kinase holoenzyme complexes, membrane microdomain, receptor complexes, transcription factor complexes, and RNA polymerase II transcription factor complexes (Figure 5(b)); and (3) molecular functions: transcription factor activity, direct ligandregulated sequence-specific DNA binding, protein phosphatase binding, and transmembrane receptor protein tyrosine kinase activity (Figure 5(c)).

3.3.2. KEGG Analysis. KEGG pathway enrichment of the top 20 pathways was identified $(P<0.05$, Figure 6(a) and Table 3), including the PI3K-Akt and IL-17 signaling pathways, and the detailed targets of the regulatory roles of this pathway are presented in Figures 6(b)-6(e). The maps of the KEGG pathway showed that the most related targets of regulation roles were AKT, MMP9, IL-6, CCND1, BCL2, MTOR, and MDM2. 
TABLE 1: The information of 743 candidate compounds in the Zhishi-Baizhu herb pair.

\begin{tabular}{|c|c|c|c|c|c|}
\hline Herb & Mol ID & Molecule name & $\mathrm{OB}(\%)$ & $\mathrm{DL}$ & Target amount \\
\hline $\mathrm{ZS}$ & MOL013277 & Isosinensetin & 51.15 & 0.44 & 27 \\
\hline ZS & MOL013279 & 5,7,4'-Trimethylapigenin & 39.83 & 0.3 & 16 \\
\hline ZS & MOL013428 & Isosakuranetin-7-rutinoside & 41.24 & 0.72 & 1 \\
\hline ZS & MOL013430 & Prangenin & 43.6 & 0.29 & 3 \\
\hline ZS & MOL013433 & Prangenin hydrate & 72.63 & 0.29 & 1 \\
\hline ZS & MOL013435 & Poncimarin & 63.62 & 0.35 & 4 \\
\hline ZS & MOL013436 & Isoponcimarin & 63.28 & 0.31 & 4 \\
\hline ZS & MOL013437 & 6-Methoxyaurapten & 31.24 & 0.3 & 11 \\
\hline ZS & MOL013440 & Citrusin B & 40.8 & 0.71 & 1 \\
\hline ZS & MOL001798 & Neohesperidin_qt & 71.17 & 0.27 & 7 \\
\hline ZS & MOL001803 & Sinensetin & 50.56 & 0.45 & 21 \\
\hline ZS & MOL001941 & Ammidin & 34.55 & 0.22 & 8 \\
\hline ZS & MOL002914 & Eriodyctiol (flavanone) & 41.35 & 0.24 & 8 \\
\hline ZS & MOL004328 & Naringenin & 59.29 & 0.21 & 37 \\
\hline ZS & MOL005100 & $\begin{array}{l}\text { 5,7-Dihydroxy-2-(3-hydroxy-4-methoxyphenyl) } \\
\text { chroman-4-one }\end{array}$ & 47.74 & 0.27 & 10 \\
\hline ZS & MOL005828 & Nobiletin & 61.67 & 0.52 & 35 \\
\hline ZS & MOL005849 & Didymin & 38.55 & 0.24 & 13 \\
\hline ZS & MOL000006 & Luteolin & 36.16 & 0.25 & 57 \\
\hline ZS & MOL007879 & Tetramethoxyluteolin & 43.68 & 0.37 & 32 \\
\hline ZS & MOL009053 & $\begin{array}{c}\text { 4-[(2S,3R)-5-[(E)-3-Hydroxyprop-1-enyl]-7-methoxy-3-methylol-2,3- } \\
\text { dihydrobenzofuran-2-yl]-2-methoxy-phenol }\end{array}$ & 50.76 & 0.39 & 11 \\
\hline $\mathrm{BZ}$ & MOL000020 & 12-Senecioyl-2E,8E,10E-atractylentriol & 62.4 & 0.22 & 184 \\
\hline $\mathrm{BZ}$ & MOL000021 & 14-Acetyl-12-senecioyl-2E, $8 \mathrm{E}, 10 \mathrm{E}$-atractylentriol & 60.31 & 0.31 & 114 \\
\hline $\mathrm{BZ}$ & MOL000022 & 14-Acetyl-12-senecioyl-2E,8Z,10E-atractylentriol & 63.37 & 0.3 & 115 \\
\hline $\mathrm{BZ}$ & MOL000028 & $\alpha$-Amyrin & 39.51 & 0.76 & 1 \\
\hline BZ & MOL000033 & $\begin{array}{l}\text { (3S,8S,9S,10R,13R,14S,17R)-10,13-Dimethyl-17-[(2R,5S)-5-propan-2-yloctan-2-yl]- } \\
2,3,4,7,8,9,11,12,14,15,16,17 \text {-dodecahydro-1H-cyclopenta[a]phenanthren-3-ol }\end{array}$ & 36.23 & 0.78 & 1 \\
\hline $\mathrm{BZ}$ & MOL000049 & $3 \beta$-Acetoxyatractylone & 54.07 & 0.22 & 16 \\
\hline $\mathrm{BZ}$ & MOL000072 & $8 \beta$-Ethoxyatractylenolide III & 35.95 & 0.21 & 5 \\
\hline
\end{tabular}

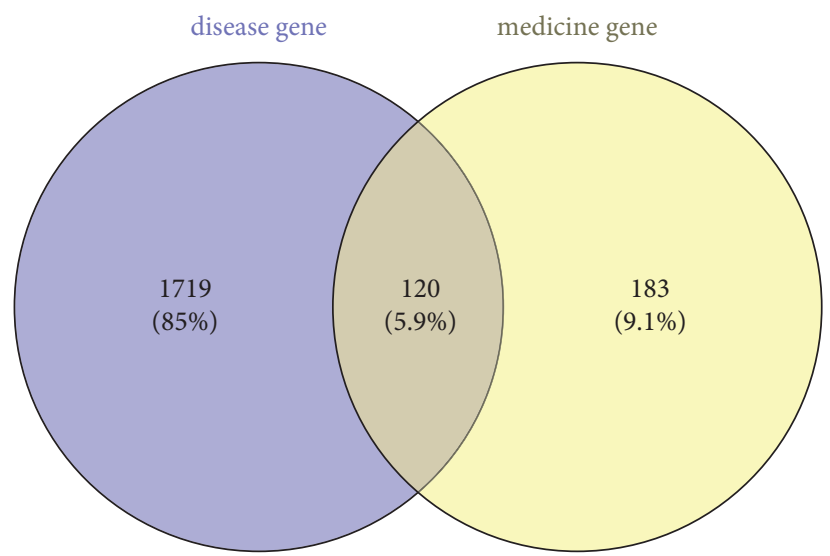

Figure 1: Venn diagram of the Zhishi-Baizhu (ZS-BZ) herb pair (medicine genes) and gastric cancer (disease genes) intersection targets. The 120 overlapping targets represent the candidate targets of the ZS-BZ herb pair against gastric cancer.

\section{Results of Molecular Docking}

We chose two main active compounds, luteolin and naringenin, which have a strong link with gastric cancer $[42,43]$, and molecular docking was used to verify the regulation of these two compounds in the top 20 selected target proteins. The results showed that most target proteins had a strong affinity for luteolin and naringenin. Except for CREB1 (PDB ID: 1ci6), the binding energies with luteolin and naringenin are -4.21 and $-4.39 \mathrm{kcal} /$ mol, respectively, whereas the other target proteins are all less than $-5 \mathrm{kcal} / \mathrm{mol}$. The molecular binding energies with luteolin and naringenin of the top 20 key target proteins are shown in Table 4, and the docking conformations of most related targets of regulation roles selected by KEGG analysis, such as AKT, MMP9, IL-6, CCND1, BCL2, MTOR, and MDM2, are shown in Figures 7(a)$7(\mathrm{n})$. 

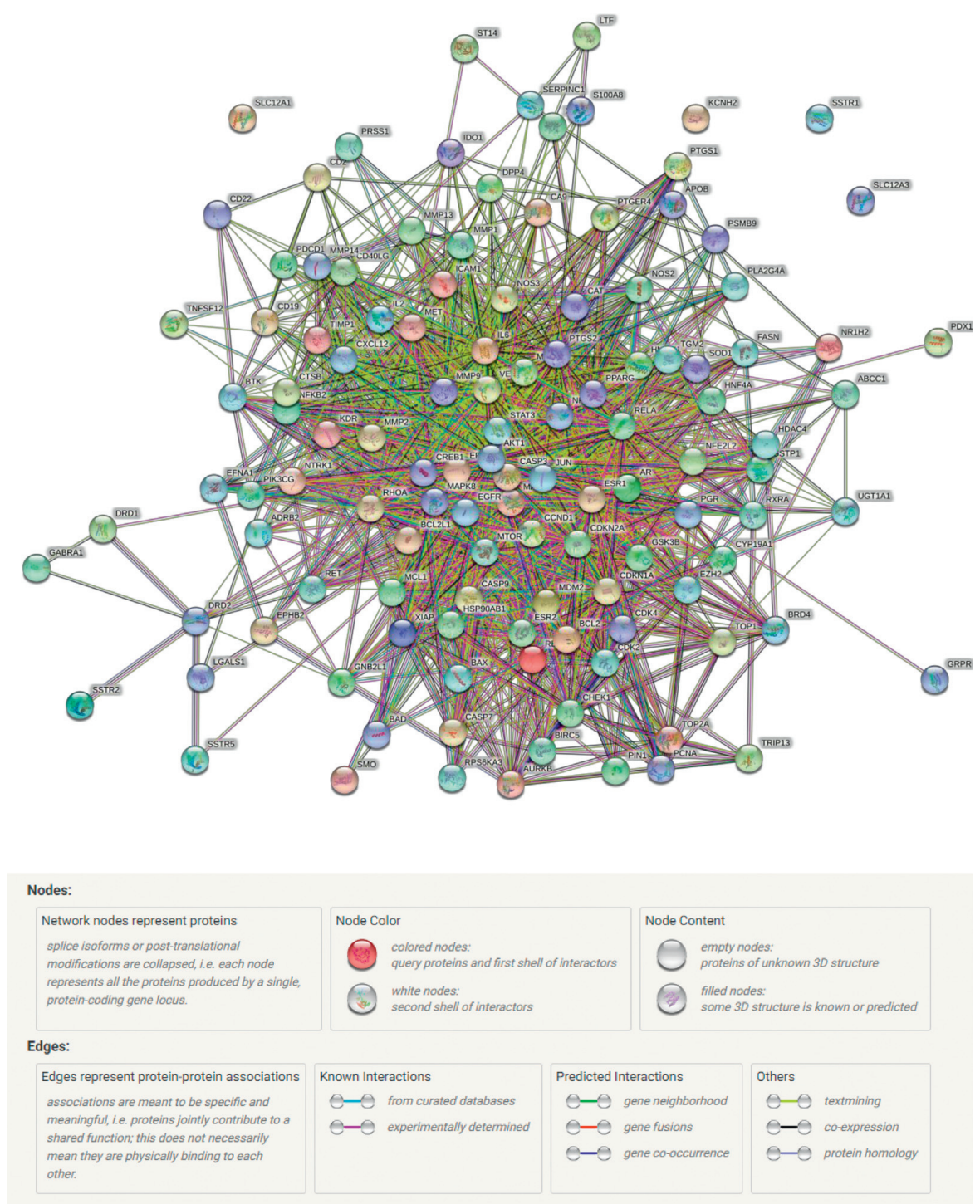

FIGURE 2: Protein-protein interaction networks of the Zhishi-Baizhu herb pair and gastric cancer.

\section{Discussion}

Traditional Chinese medicine has been used in the struggle against diseases for thousands of years. The famous traditional Chinese physician Shi Jinmo found out many effects of the ZS-BZ herb pair for treating gastrointestinal diseases while studying drug pairs. ZS has been used to promote digestion, remove food, and resolve mass between belly and abdomen, whereas BZ has been used to harmonise qi and blood. When these two herbs are combined, one for eliminating and one for tonifying, as well as one for being fast and one for slowing down, they gain mutual restraint and utilisation with each other; thus, they finally achieve the therapeutic effect of tonifying without obstructing the stagnation (the theory of traditional Chinese medicine). They also relieve oppression and masses, eliminate pathogenic factors without damaging healthy qi, and strengthen the spleen and stomach [44].
Network pharmacology and molecular docking methods were used to explore the possible pharmacological mechanisms of the ZS-BZ herb pair related to gastric cancer in this study, which indicated that 27 compounds and 120 target genes were related to gastric cancer. According to the results of GO and KEGG pathway enrichment analyses, we found that the effects of the ZS-BZ herb pair against gastric cancer may be due to the active compounds of the ZS-BZ herb pair, especially for luteolin and naringenin which could influence the regulation of the PI3K-Akt and IL-17 signaling pathway. They may also be related to the apoptotic signaling pathway, cyclin-dependent protein kinase holoenzyme complex, and receptor complex and transcription factor complex. Based on the results of PPI analysis and Cytoscape Hub, AKT1, MMP9, IL-6, CCND1, BCL2, MTOR, and MDM2 were selected as key targets, and they reflect a strong affinity with luteolin and naringenin as the results of the molecular docking method. 




Figure 3: Drug-target interaction of the Zhishi-Baizhu (ZS-BZ) herb pair and gastric cancer. Red triangle represents gastric cancer, dark blue round rectangles represent the herbs ZS and BZ, and grass green ellipses represent potential common targets of the ZS-BZ herb pair and gastric cancer.

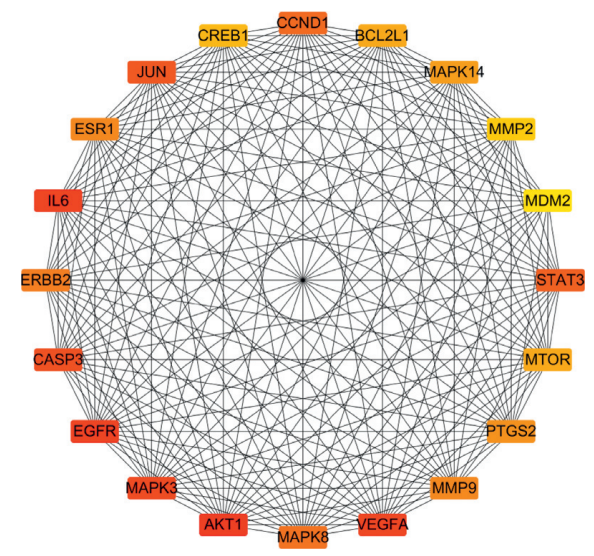

Figure 4: Top 20 key targets in PPI network.

Gastric mucosal carcinogenesis is a progressive process. The most recognized mode of intestinal gastric cancer is atrophic gastritis intestinal metaplasia dysplasia intramucosal cancer proposed by Correa [45] in 1988. The clinical symptoms of gastric cancer include epigastric pain, nausea, vomiting, anorexia, an epigastric lump, and the damage of gastric mucosal layer [46]. Recent studies have validated that these symptoms could effectively be ameliorated by the combination use of ZS and BZ. Zhizhu
Pills can effectively regulate the expression of PI3K and Akt [47]. BZ can also repair gastrointestinal mucosal injury by regulating the expression of the IL-17 signaling pathway [48]; in our study, the PI3K-Akt and IL-17 signaling pathways are two key pathways involved in gastric cancer.

The PI3K-Akt signaling pathway is significant in the formation and progression of many malignancies, and it has become a hot spot as a therapeutic target for cancer. It 
TABLE 2: Key targets in protein-protein interaction network which are greater than the average value (top 20).

\begin{tabular}{lcc}
\hline Rank & Name & Degree \\
\hline 1 & AKT1 & 85 \\
2 & EGFR & 75 \\
2 & IL-6 & 75 \\
4 & VEGFA & 74 \\
5 & MAPK3 & 73 \\
6 & CASP3 & 72 \\
7 & JUN & 70 \\
8 & STAT3 & 69 \\
9 & CCND1 & 67 \\
10 & MAPK8 \\
11 & ERBB2 & 66 \\
12 & ESR1 & 61 \\
12 & MMP9 & 60 \\
14 & PTGS2 & 60 \\
15 & MAPK14 & 58 \\
16 & BCL2L1 & 56 \\
16 & MTOR & 53 \\
18 & CREB1 & 53 \\
20 & MMP2 & 50 \\
\hline
\end{tabular}

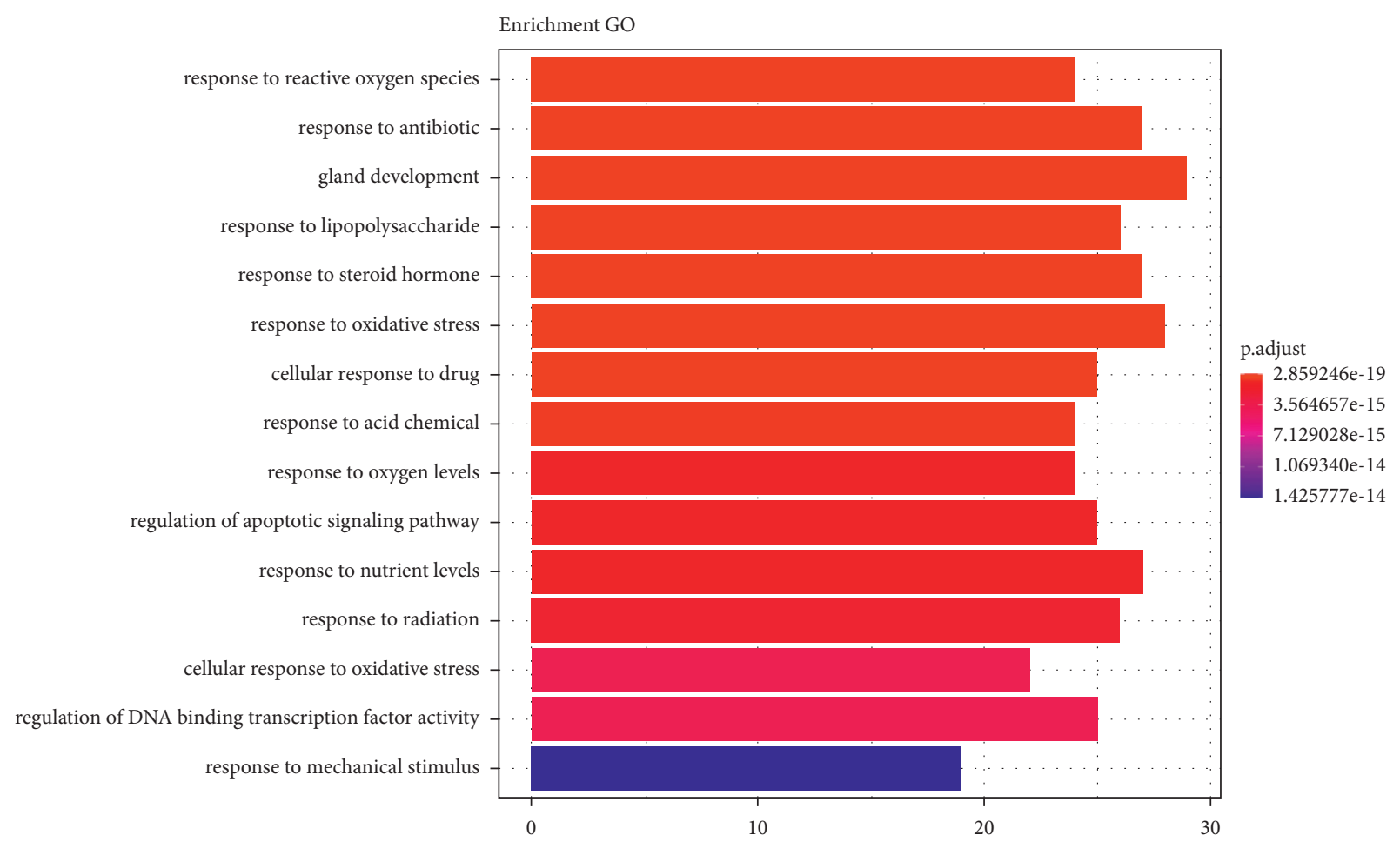

(a)

Figure 5: Continued. 


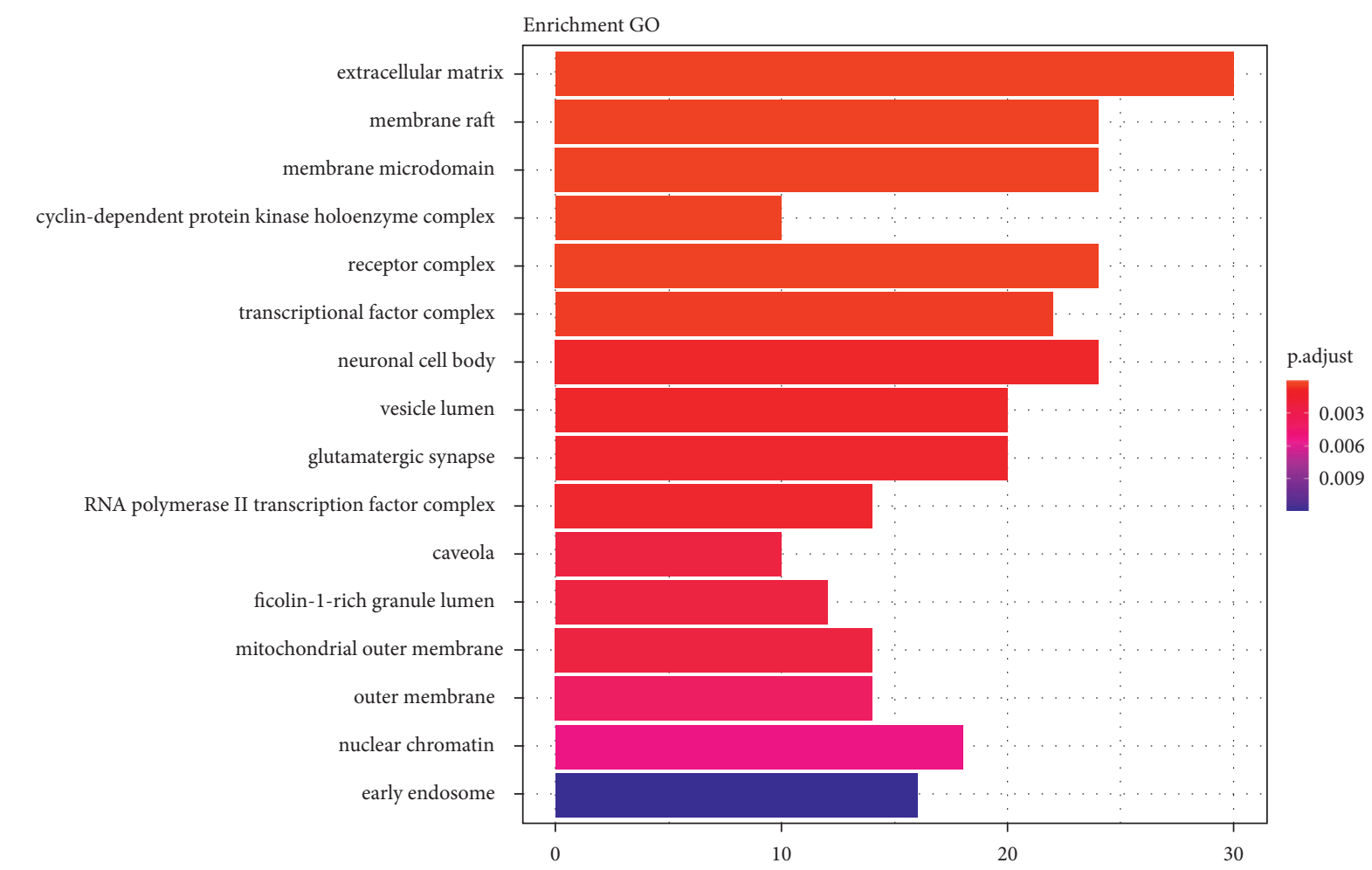

(b)

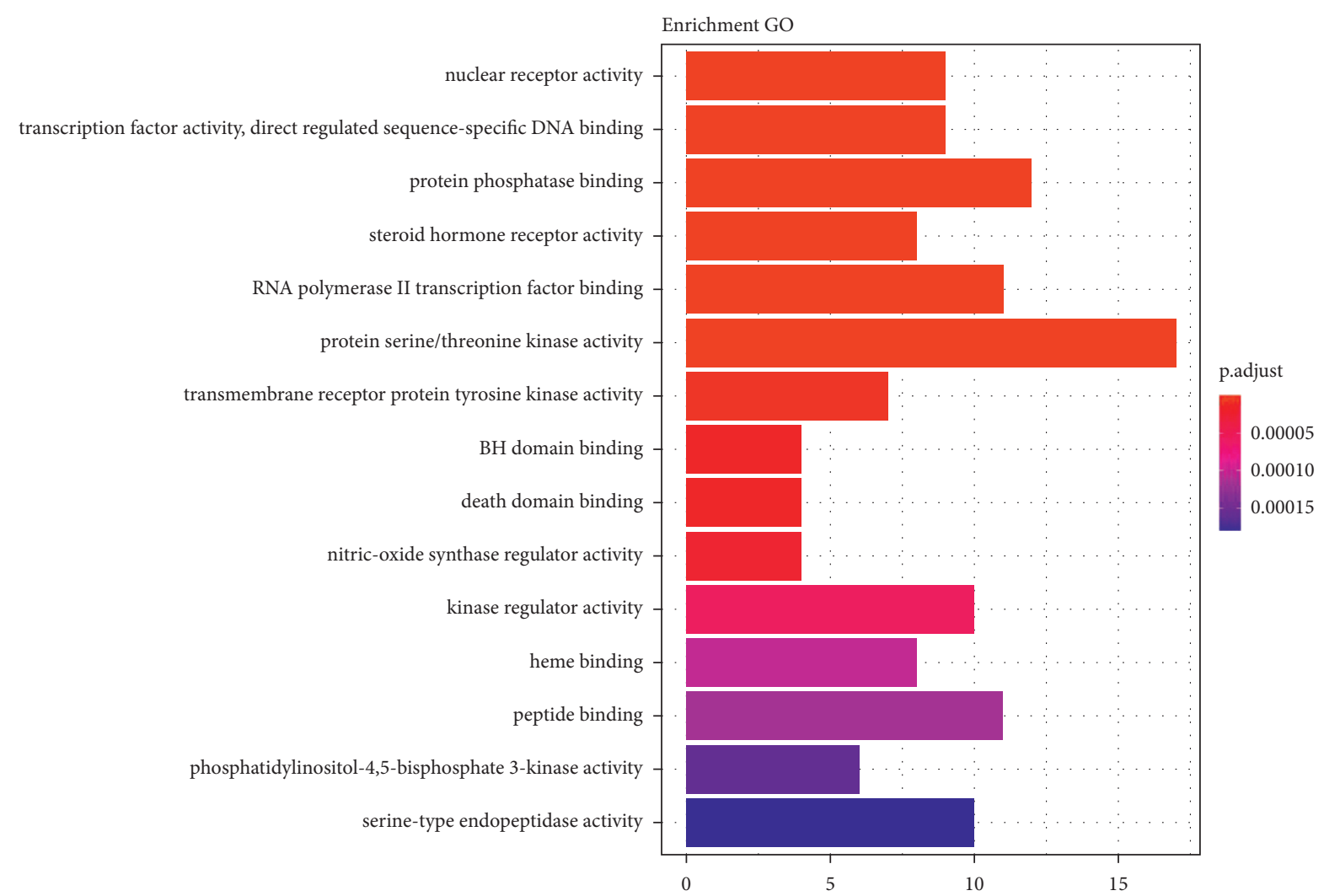

(c)

FIGURE 5: (a) Results of biological process (top 15). (b) Results of cellular components (top 16). (c) Results of molecular function (top 15). 


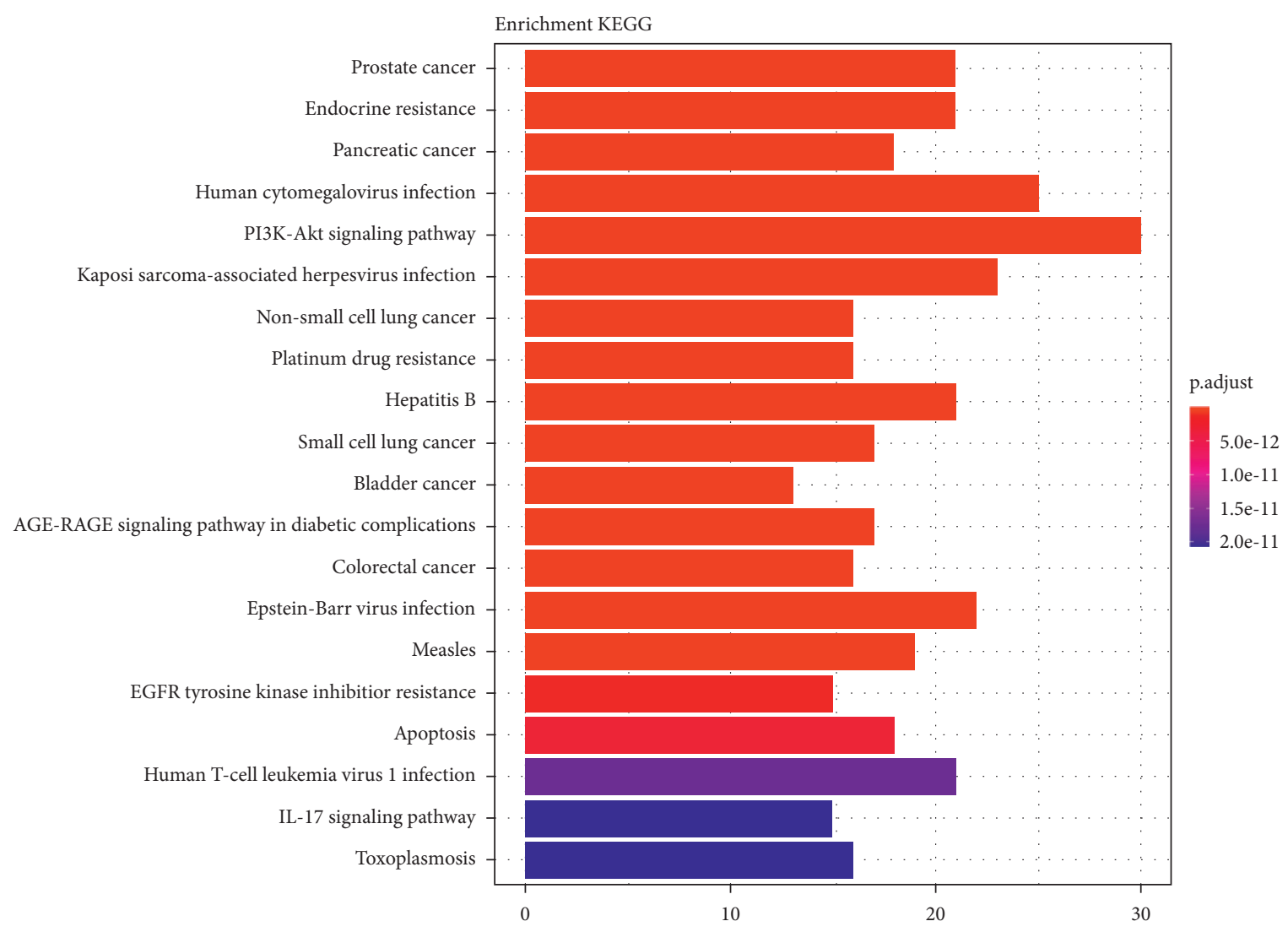

(a)

Figure 6: Continued. 


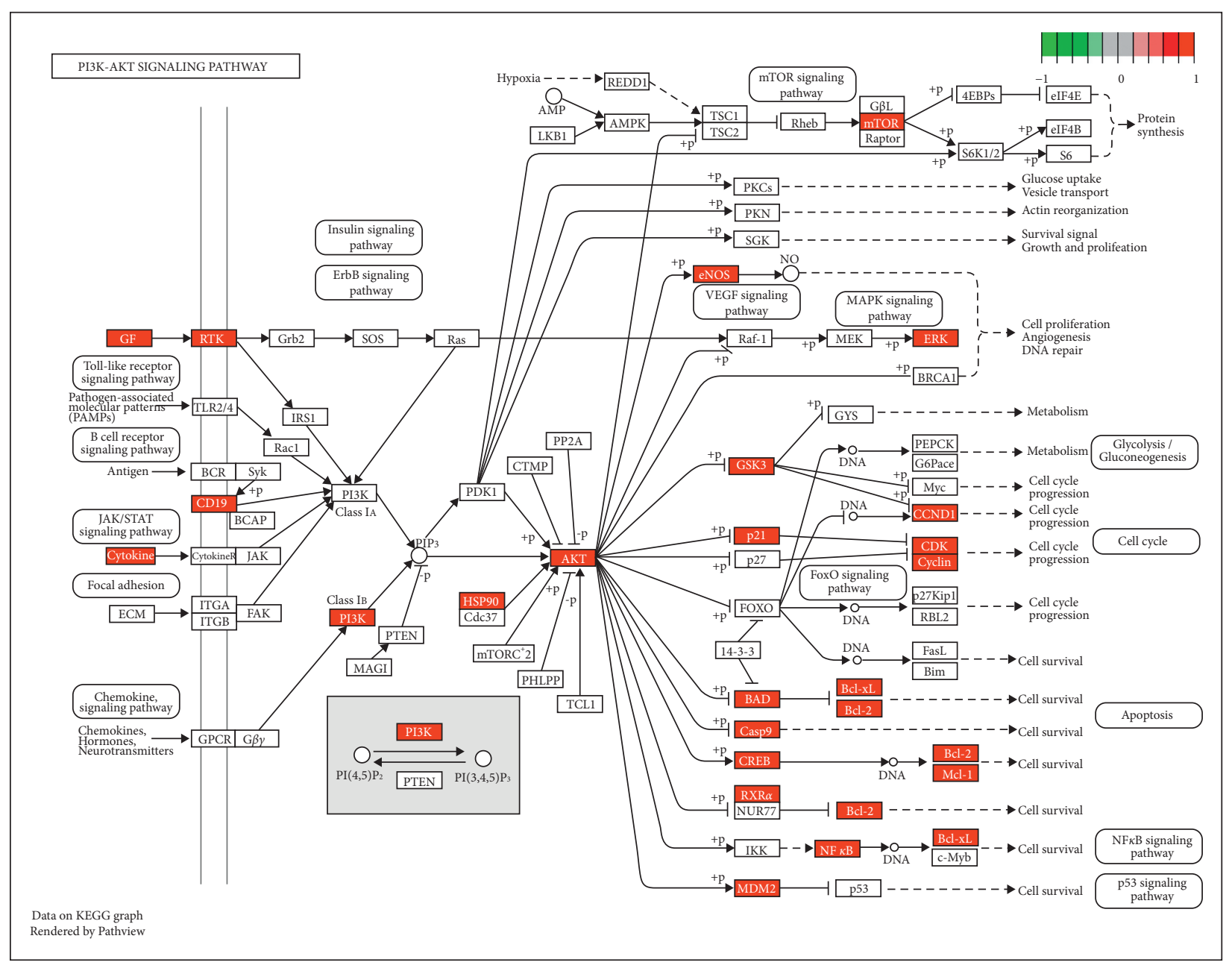

(b)

Figure 6: Continued. 


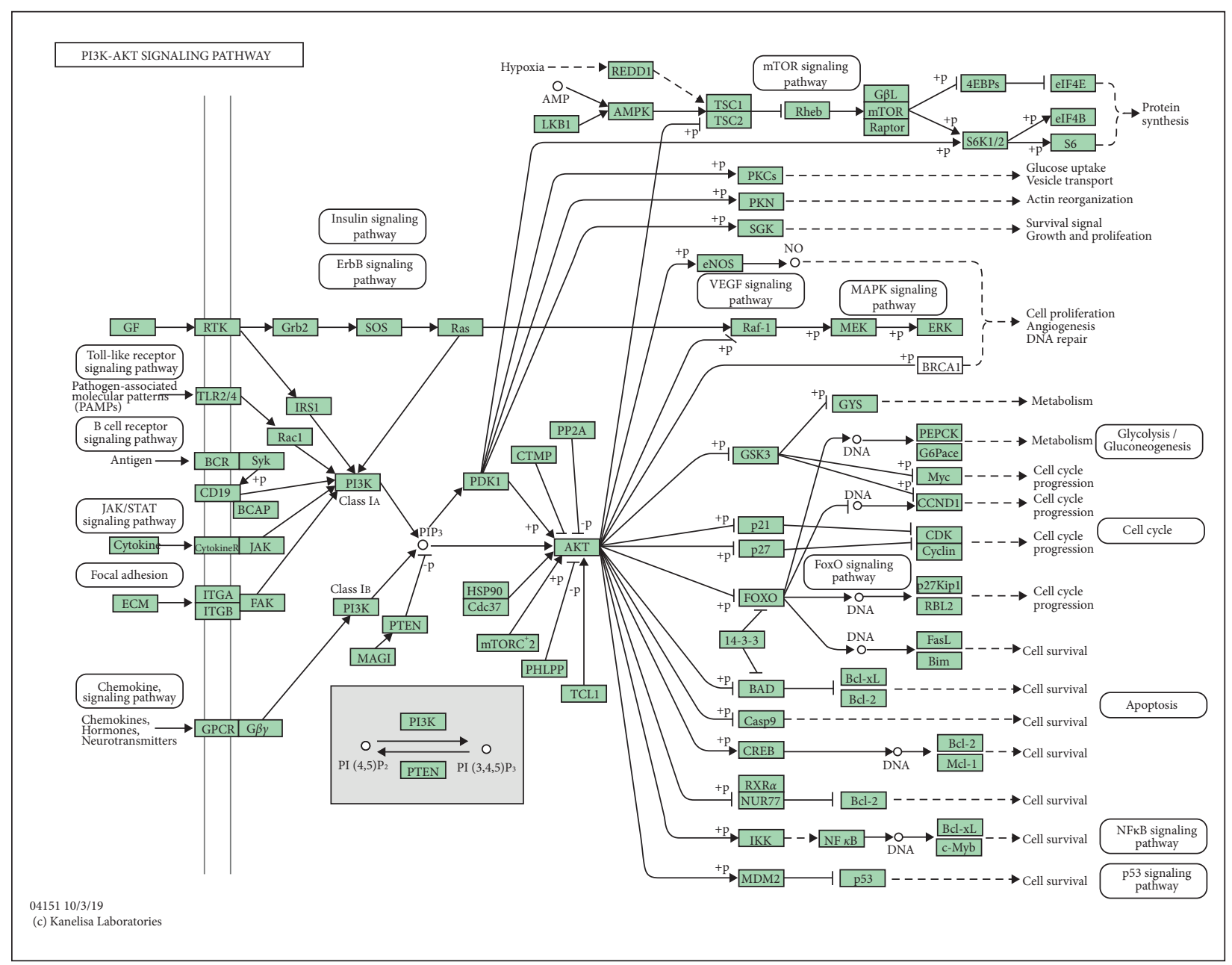

(c)

Figure 6: Continued. 


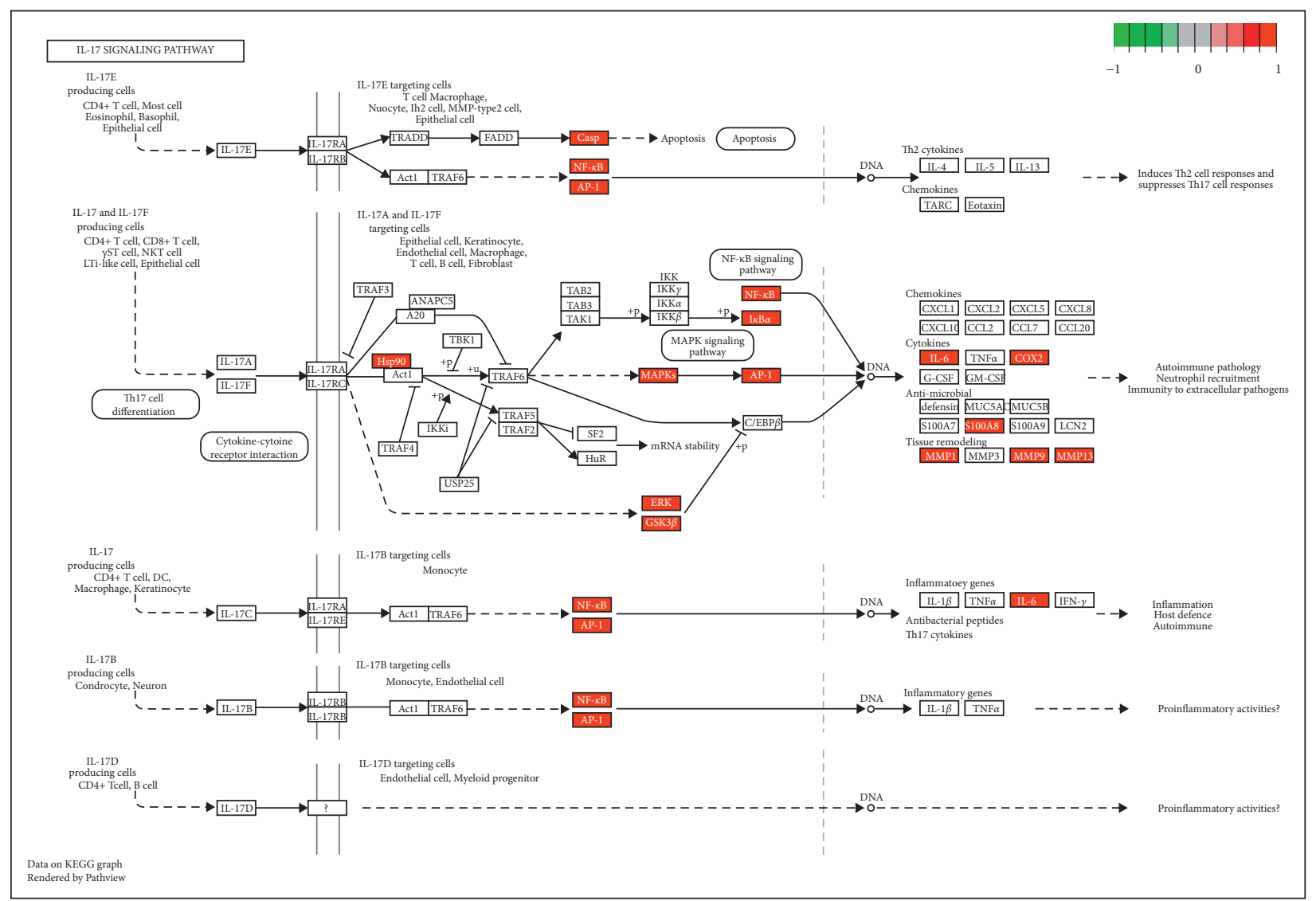

(d)

FIgURE 6: Continued. 


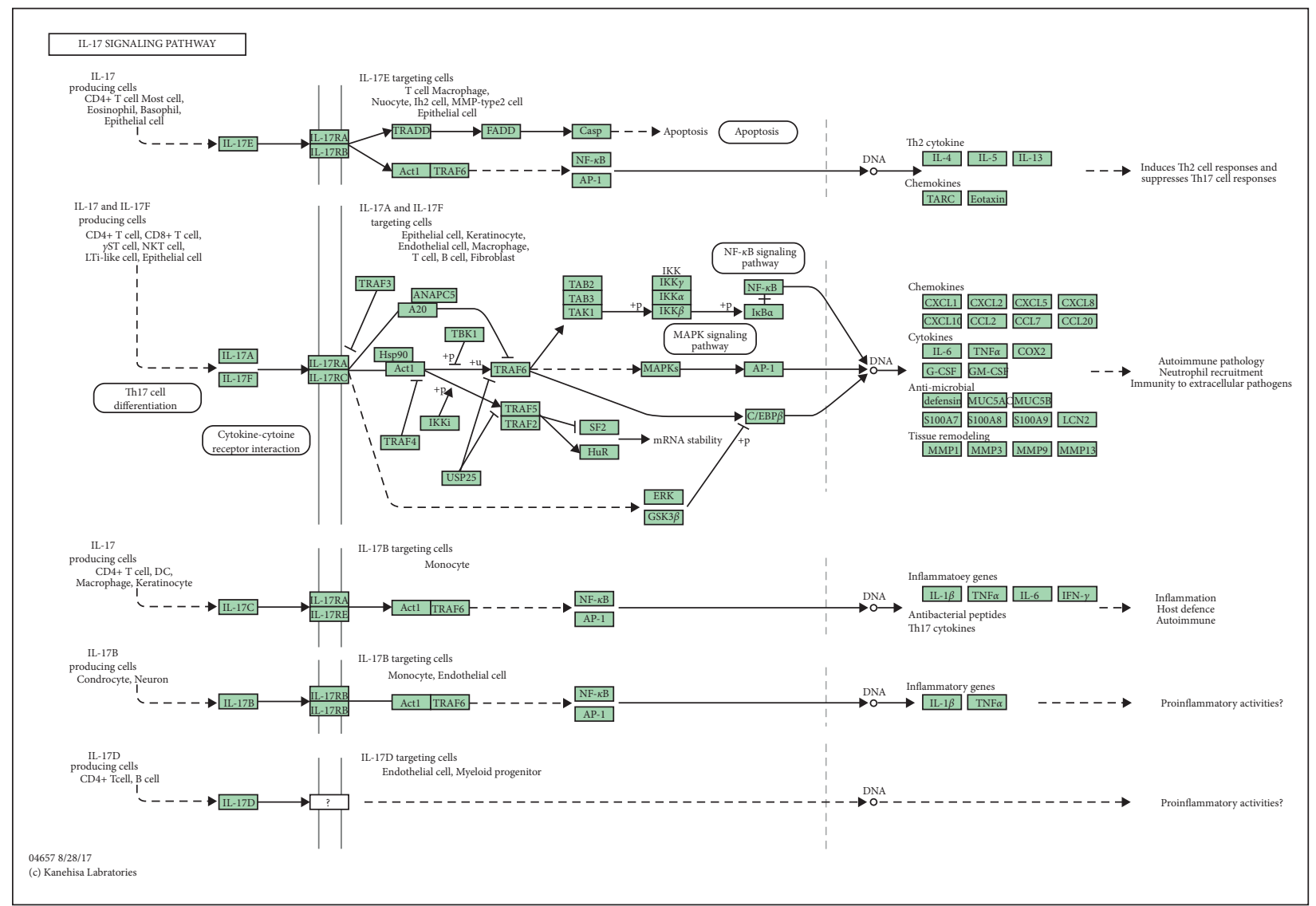

(e)

FIGURE 6: (a) Results of KEGG enrichment analysis (top 20). (b) KEGG pathway analysis of PI3K-Akt signaling pathway of the ZhishiBaizhu herb pair in the regulation of gastric cancer. Red rectangles represent the targets of regulation roles. (c) KEGG pathway analysis of the PI3K-Akt signaling pathway of the Zhishi-Baizhu herb pair in the regulation of gastric cancer. Green rectangles represent the targets of potential roles. (d) KEGG pathway analysis of IL-17 signaling pathway of Zhishi-Baizhu herb pair in the regulation of gastric cancer. Red rectangles represent the targets of regulation roles. (e) KEGG pathway analysis of IL-17 signaling pathway of Zhishi-Baizhu herb pair in the regulation of gastric cancer. Green rectangles represent the targets of potential roles.

TABLE 3: Results of KEGG enrichment analysis (top 20).

\begin{tabular}{|c|c|c|c|c|}
\hline ID & Description & $P$ value & $P$ adjusted & Count \\
\hline hsa05215 & Prostate cancer & $4.71 E-20$ & $7.21 E-18$ & 21 \\
\hline hsa01522 & Endocrine resistance & $5.93 E-20$ & $7.21 E-18$ & 21 \\
\hline hsa05212 & Pancreatic cancer & $4.76 E-18$ & $3.85 E-16$ & 18 \\
\hline hsa05163 & Human cytomegalovirus infection & $1.99 E-16$ & $1.16 E-14$ & 25 \\
\hline hsa04151 & PI3K-Akt signalling pathway & $2.39 E-16$ & $1.16 E-14$ & 30 \\
\hline hsa05167 & Kaposi's sarcoma-associated herpesvirus infection & $7.92 E-16$ & $3.21 E-14$ & 23 \\
\hline hsa05223 & Non-small cell lung cancer & $1.15 E-15$ & $4.00 E-14$ & 16 \\
\hline hsa01524 & Platinum drug resistance & $1.46 E-15$ & $4.44 E-14$ & 16 \\
\hline hsa05161 & Hepatitis B & $2.96 E-15$ & $7.99 E-14$ & 21 \\
\hline hsa05222 & Small cell lung cancer & $3.78 E-15$ & $8.53 E-14$ & 17 \\
\hline hsa05219 & Bladder cancer & $3.86 E-15$ & $8.53 E-14$ & 13 \\
\hline hsa04933 & AGE-RAGE signaling pathway in diabetic complications & $1.63 E-14$ & $3.30 E-13$ & 17 \\
\hline hsa05210 & Colorectal cancer & $2.32 E-14$ & $4.26 E-13$ & 16 \\
\hline hsa05169 & Epstein-Barr virus infection & $2.46 E-14$ & $4.26 E-13$ & 22 \\
\hline hsa05162 & Measles & $2.63 E-14$ & $4.26 E-13$ & 19 \\
\hline hsa01521 & EGFR tyrosine kinase inhibitor resistance & $1.16 E-13$ & $1.75 E-12$ & 15 \\
\hline hsa04210 & Apoptosis & $2.38 E-13$ & $3.41 E-12$ & 18 \\
\hline hsa05166 & Human T-cell leukaemia virus 1 infection & $1.29 E-12$ & $1.74 E-11$ & 21 \\
\hline hsa04657 & IL-17 signaling pathway & $1.68 E-12$ & $2.07 E-11$ & 15 \\
\hline hsa05145 & Toxoplasmosis & $1.70 E-12$ & $2.07 E-11$ & 16 \\
\hline
\end{tabular}


TABLE 4: The binding energies of top 20 key target proteins with luteolin and naringenin.

\begin{tabular}{lccc}
\hline Target & PDB ID & Luteolin $(\mathrm{kcal} / \mathrm{mol})$ & Naringenin $(\mathrm{kcal} / \mathrm{mol})$ \\
\hline AKT1 & $4 \mathrm{gah}$ & -7.45 & -6.18 \\
EGFR & $4 \mathrm{qvx}$ & -6.42 & -5.83 \\
IL-6 & $6 \mathrm{mg}^{3}$ & -6.86 & -5.51 \\
VEGFA & $6 \mathrm{v} 7 \mathrm{k}$ & -6.03 & -6.63 \\
MAPK3 & $4 \mathrm{qtb}$ & -6.52 & -5.2 \\
CASP3 & $4 \mathrm{jqy}$ & -6.16 & -6.07 \\
JUN & $3 \mathrm{v} 3 \mathrm{v}$ & -6.2 & -6.06 \\
STAT3 & $6 \mathrm{smb}$ & -7.01 & -5.05 \\
CCND1 & $2 \mathrm{w} 9 \mathrm{f}$ & -6.84 & -5.86 \\
MAPK8 & $3 \mathrm{om}$ & -5.77 & -5.04 \\
ERBB2 & $4 \mathrm{hrn}$ & -5.1 & -5.41 \\
ESR1 & $1 \mathrm{a} 52$ & -5.42 & -5.77 \\
MMP9 & $116 \mathrm{j}$ & -6.78 & -6.45 \\
PTGS2 & $5 \mathrm{ikr}$ & -6.36 & -5.8 \\
MAPK14 & $5 \mathrm{omh}$ & -7.03 & -6.77 \\
BCL2L1 & $4 \mathrm{qvx}$ & -7.59 & -7.41 \\
MTOR & $3 \mathrm{qar}$ & -6.36 & -5.35 \\
CREB1 & $1 \mathrm{ci} 6$ & -4.21 & -4.39 \\
MMP2 & $1 \mathrm{rtg}$ & -6.67 & -6.06 \\
MDM2 & $3 \mathrm{lbk}$ & -5.78 & -5.79 \\
\hline
\end{tabular}

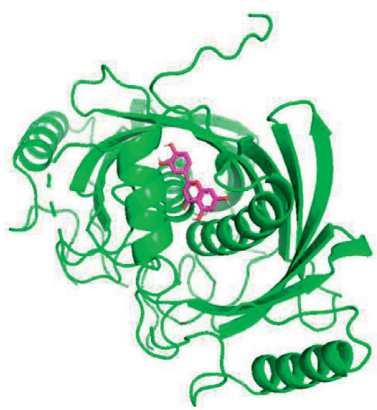

(a)

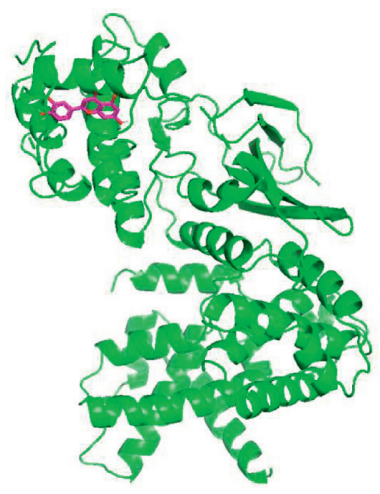

(d)

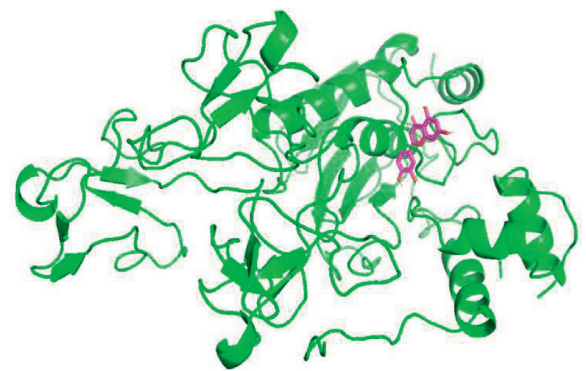

(b)

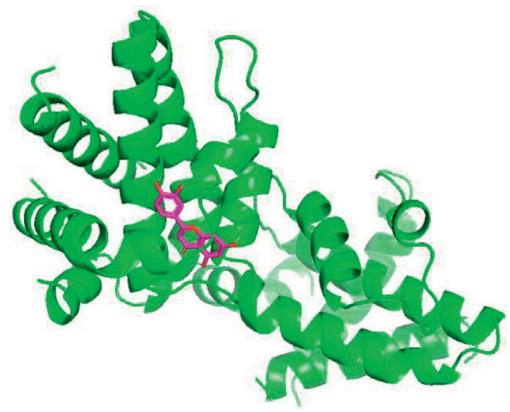

(e)

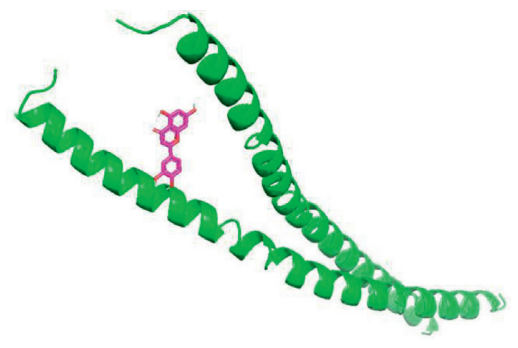

(c)

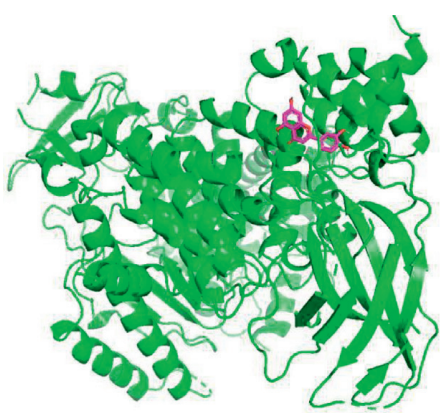

(f)

Figure 7: Continued. 


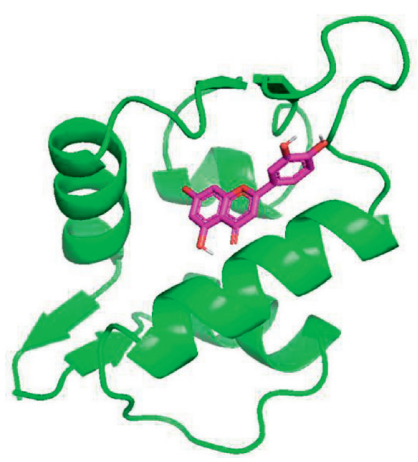

(g)

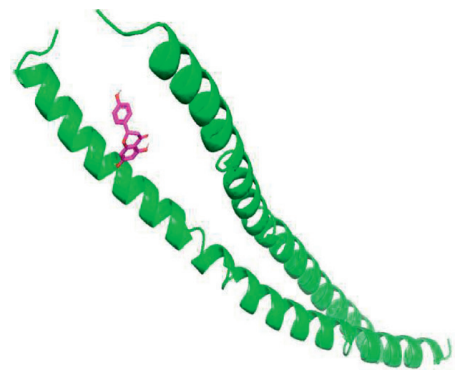

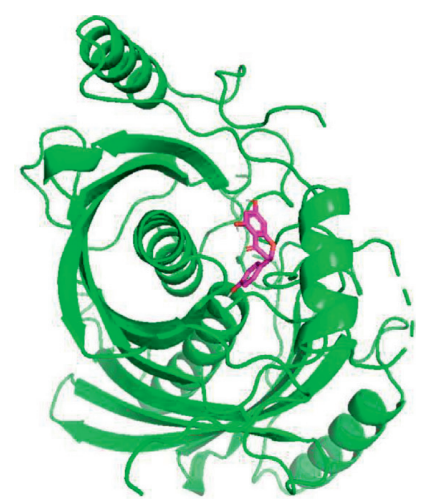

(h)

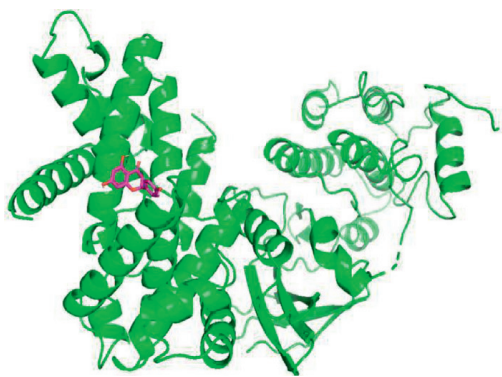

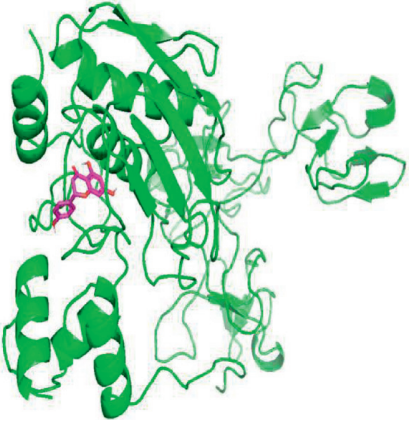

(i)

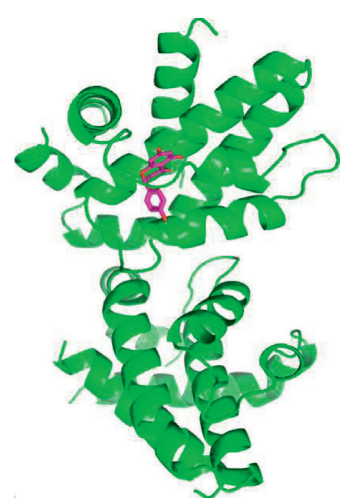

(l) (j)

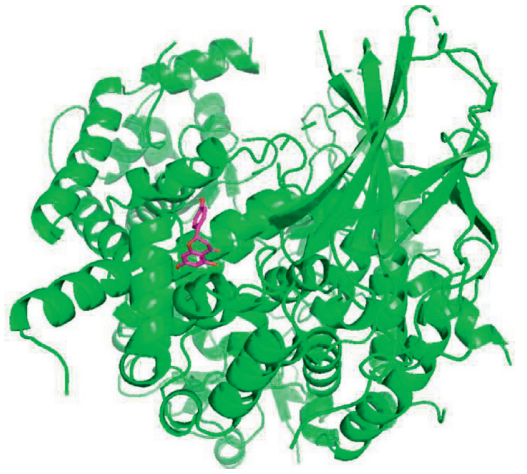

(m) (k)

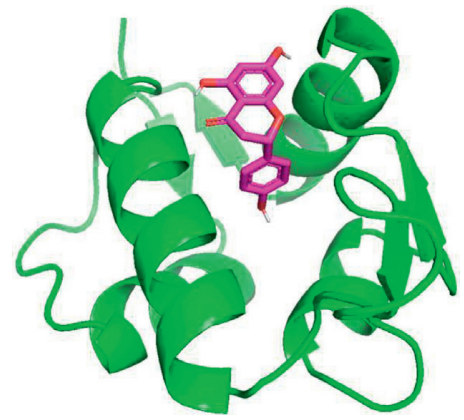

(n)

FIgURE 7: (a) Luteolin with AKT1. (b) Luteolin with MMP9. (c) Luteolin with IL-6. (d) Luteolin with CCND1. (e) Luteolin with BCL2. (f) Luteolin with MTOR. (g) Luteolin with MDM2. (h) Naringenin with AKT1. (i) Naringenin with MMP9. (j) Naringenin with IL-6. (k) Naringenin with CCND1. (l) Naringenin with BCL2. (m) Naringenin with MTOR. (n) Naringenin with MDM2.

involves the regulation of tumour cell of gastric cancer survival, proliferation, invasion, and migration $[49,50]$. Various studies have indicated that the PI3K-AKT signaling pathway is associated with tumour resistance to chemotherapy and radiotherapy [51].

The experiments showed the overexpression of AKT1 and MTOR genes in gastric mucosa of gastric cancer patients and showed that they play vital roles in cell growth, proliferation, metabolism, angiogenesis, and survival in mediating cellular functions [52]. Furthermore, mTOR is also involved in regulating the synthesis of biological macromolecules, such as nucleotides, proteins, and lipids. Another study showed that the metastasis of gastric cancer cells was induced by MMP9, and this process was activated by the PI3K-AKT signaling pathway. A further study found that the blockade of the PI3K-AKT signaling pathway by using MMP9 proenzyme inhibitor could suppress the distant metastasis of gastric cancer cells [53].

It has been reported that IL-6 is highly upregulated in many cancers and is one of the most important cytokine families in tumorigenesis and metastasis [54] and is highly expressed in gastric cancer tissues [55]. It can effectively control the pathological changes of cancer by mediating the expression of IL-6 in the IL-17 signaling pathway [56]. In addition, CCND1 is an important target for cell proliferation in gastric cancer [57], and it has been shown that high 
CCND1 expression levels are closely related to shorter survival rates of gastric cancer patients. It has been found in tissues of gastric cancer that the upregulated expression of BCL2 predicted a poor prognosis [58].

Some scientific tests have established that luteolin is an active component of ZS, which is a potential chemotherapeutic agent against gastric cancer. It can suppress cell cycle arrest, proliferation, colony formation, and accelerate apoptosis in gastric cancer cells in vitro and in vivo by regulating the PI3K and AKT signaling pathways [59]. In addition, studies have substantiated that luteolin can significantly inhibit the IL-17 signaling pathway in digestive tract disease [60] and then decrease the level of IL- 6 in the patient's serum.

Naringenin induces apoptosis of gastric cancer SGC7901 cells by decreasing the expression of MMP9 by downregulating the AKT pathway [61]. It can promote the apoptosis of human gastric cancer SGC7901 cells by inhibiting the PI3K-Akt signaling pathway and plays a tumour suppressor effect, providing a proper therapeutic target for the treatment of gastric cancer [62]. In our study, we preliminarily explored the mechanism of the ZS-BZ herb pair in treating gastric cancer by using network pharmacology and molecular docking. We found that there is a close link with the regulation of the PI3K-Akt and IL-17 signaling pathway with luteolin and naringenin. AKT1, MMP9, IL-6, CCND1, BCL2, MTOR, and MDM2 were found to be the seven key targets in these two signaling pathways, and they reflect a strong affinity with luteolin and naringenin.

\section{Conclusions}

In summary, the results of our study showed that the ZS-BZ herb pair may play a therapeutic role in treating gastric cancer based on the active compounds luteolin and naringenin. These two compounds mainly act on seven targets, namely, AKT1, MMP9, IL-6, CCND1, BCL2, MTOR, and MDM2; they may be related to the regulation of the PI3KAkt signaling pathway and IL-17 signaling pathway. Our efforts have provided evidence and we have obtained a good understanding of the multitarget, multicompound, and multipathway synergy of traditional Chinese medicine in treating gastric cancer. However, in vivo and in vitro experiments and clinical studies should be conducted to verify the mechanism of the ZS-BZ herb pair against gastric cancer.

\section{Data Availability}

All the data and materials used in the current study are available from the corresponding author upon reasonable request.

\section{Disclosure}

The funder had no role in study design, data collection and analysis, decision to publish, or preparation of the manuscript.

\section{Conflicts of Interest}

The authors declare that there are no conflicts of interest.

\section{Authors' Contributions}

Ying Qu, Xiangyang Yang, and Jingxiang Li conceived the concept and designed the study methods. Shuxin Zhang and Lu Zhou designed the enrichment analysis. Zi Lin, Yuzhang Yin, Mengyuan Wang, and Shiying Li constructed the images and charts. Jinlong Liu and Yang Yang provided advice on the methodology and contributed to targets searching. Zhiying Wang and Nan Wang polished and revised the manuscript. All authors approved the final manuscript. Ying Qu, Zhiying Wang, and Nan Wang wrote the manuscript. Ying $\mathrm{Qu}$ and Xiangyang Yang contributed equally to this article.

\section{Acknowledgments}

The authors are really grateful for Lu Zhou's contribution to the design, enrichment analysis, and molecular docking. Beijing Municipal Natural Science Foundation supported this study (7202112).

\section{References}

[1] O. Serra, M. Galán, M. M. Ginesta, M. Calvo, N. Sala, and R. Salazar, "Comparison and applicability of molecular classifications for gastric cancer," Cancer Treatment Reviews, vol. 77, pp. 29-34, 2019.

[2] J. Yin, X. Wu, S. Li, C. Li, and Z. Guo, "Impact of environmental factors on gastric cancer: a review of the scientific evidence, human prevention and adaptation," Journal of Environmental Sciences, vol. 89, pp. 65-79, 2020.

[3] Z. Song, Y. Wu, J. Yang, D. Yang, and X. Fang, "Progress in the treatment of advanced gastric cancer," Tumor Biology, vol. 39, no. 7, 2017.

[4] S. Tsutsumi, E. Oki, S. Ida et al., "Laparoscopic gastrectomy for gastric cancer with peritoneal dissemination after induction chemotherapy," Case Reports in Gastroenterology, vol. 7, no. 3, pp. 516-521, 2013.

[5] E. U. Cidon, S. G. Ellis, S. G. Ellis et al., "Molecular targeted agents for gastric cancer: a step forward towards personalized therapy," Cancers, vol. 5, no. 1, pp. 64-91, 2013.

[6] K.-F. Liao, T.-L. Chiu, S.-Y. Huang et al., "Anti-Cancer effects of radix angelica sinensis (danggui) and N-butylidenephthalide on gastric cancer: implications for REDD1 activation and mTOR inhibition," Cellular Physiology and Biochemistry, vol. 48, no. 6, pp. 2231-2246, 2018.

[7] J. Xu, W. Shen, B. Pei et al., "Xiao Tan He Wei Decoction reverses MNNG-induced precancerous lesions of gastric carcinoma in vivo and vitro: regulation of apoptosis through NF- $\kappa$ B pathway," Biomedicine \& Pharmacotherapy, vol. 108, pp. 95-102, 2018.

[8] Y. Hu, X. Pan, M. Nie et al., "A clinical study of Yiqi Huayu Jiedu decoction reducing the risk of postoperative gastric cancer recurrence and metastasis," Medicine, vol. 99, no. 33, Article ID e21775, 2020.

[9] L. Zhao, A.-G. Zhao, G. Zhao et al., "Survival benefit of traditional Chinese herbal medicine (a herbal formula for invigorating spleen) in gastric cancer patients with peritoneal metastasis," Evidence-based Complementary and Alternative Medicine, vol. 2014, Article ID 625493, 6 pages, 2014.

[10] J. R. Hu, Y. Q. Duan, and X. P. Liu, "Review on traditional Chinese medicine treatment for gastric cancer," Liaoning J Tradit Chin Med, vol. 47, no. 8, pp. 198-202, 2020, in Chinese. 
[11] X. J. Wang, Z. X. Wang, and B. C. Wu, "National famous TCM doctor WANG xingkuan's experience in treating stomach cancer from "Stasis-Toxin"," J Hunan Univ Chin Med, vol. 40, no. 10, pp. 1249-1252, 2020, in Chinese.

[12] Y. T. Chen, X. Zhu, and Y. Z. Chen, "Progress in the study and application of zhizhu pills and its derivate prescriptions," J Jiangxi Univ Tradit Chin Med, vol. 31, no. 6, pp. 108-112, 2019, in Chinese.

[13] M. Liu and D. C. Wang, "The treatment of zhishi ershu kangai decoction on 20 cases," Heilongjiang J Tradit Chin Med, vol. 4, p. 25, 2004, in Chinese.

[14] S. C. Zuo and H. J. Li, "Experience of QI yuanfu in treating gastric cancer by zhiqiao and baizhu," Shandong Journal of Traditional Chinese Medicine, vol. 36, no. 4, pp. 313-314, 2017, in Chinese.

[15] L. Y. Zhang and M. X. Liang, "Exploitation and utilization of potential function in immature orange fruit," Chinese Journal of Traditional Chinese Medicine Pharmacy, vol. 31, no. 7, pp. 2789-2792, 2016, in Chinese.

[16] L. F. Wang, X. L. Liu, and H. T. LI, "Mechanism of Aurantii Fructus Immaturus volatile oil in treatment of slow transit constipation based on network pharmacology," Zhongguo Zhongyao Zazhi, vol. 45, no. 8, pp. 1909-1917, 2020, in Chinese.

[17] Z. H. Zhao and Y. Li, "Analysis on function of "removing cold and heat condensation" in treatise on febrile diseases," Acta Chin Med Pharm, vol. 34, no. 10, pp. 2100-2102, 2019, in Chinese.

[18] X. X. Zhang, Z. Y. Li, and Y. L. Ma, "Progress in research of traditional Chinese medicine Citrus aurantium," Zhongguo Zhongyao Zazhi, vol. 40, no. 2, pp. 185-190, 2015, in Chinese.

[19] Y. Dong, G. Ji, and A. L. Cao, "Effects of sinensetin on proliferation and apoptosis of human gastric cancer AGS cells," Zhongguo Zhongyao Zazhi, vol. 36, no. 6, pp. 790-794, 2011, in Chinese.

[20] Chinese Pharmacopoeia Commission, Pharmacopoeia of the People's Republic of China 2015, vol. 1, China Medical Science Press, Beijing, China, in Chinese.

[21] X. J. Zhang and D. D. Zuo, "The new research progress on chemical constituents and pharmacological effects of atractylodes macrocephala koidz," Traditional Chinese Medicine, vol. 35, no. 6, pp. 101-106, 2018, in Chinese.

[22] J. Wang, S. Y. Zhang, and Y. C. Sheng, "Research progress on pharmacological actionof baizhu in treatment of gastrointestinal diseases," Chin Archives Tradit Chin Med, vol. 36, no. 12 , pp. 2854-2858, 2018, in Chinese.

[23] Y. T. Shen, R. Y. Que, and L. B. Lin, "Clinic application of Zhizhu pill," Journal of Changchun University of Traditional Chinese Medicine, vol. 32, no. 5, pp. 926-928, 2016, in Chinese.

[24] F. Zhao, D. C. Tang, and S. Zhang, "Effect of zhizhu granules on expressions of VEGF and COX-2 in rat model with chronic atrophic gastritis," Chinese Journal of Experimental Traditional Medical Formulae, vol. 22, no. 24, 2016, in Chinese.

[25] S. L. Qiu, S. N. Ding, and X. Pang, "Progress in the diagnosis and treatment of gastric cancer with traditional Chinese medicine," Zhejiang Journal of Integrated Traditional Chinese and Western Medicine, vol. 30, no. 7, pp. 602-604, 2020, in Chinese.

[26] S. Sheng, Z.-X. Yang, F.-Q. Xu, and Y. Huang, "Network pharmacology-based exploration of synergistic mechanism of guanxin II formula (冠心II号方) for coronary heart disease," Chinese Journal of Integrative Medicine, vol. 27, no. 2, pp. 106-114, 2021.
[27] W. Ren, Z. Luo, F. Pan et al., "Integrated network pharmacology and molecular docking approaches to reveal the synergistic mechanism of multiple components in Venenum Bufonis for ameliorating heart failure," PeerJ, vol. 8, Article ID e10107, 2020.

[28] C. Li, X. Du, Y. Liu et al., "A systems pharmacology approach for identifying the multiple mechanisms of action for the rougui-fuzi herb pair in the treatment of cardiocerebral vascular diseases," Evidence-based Complementary and Alternative Medicine, vol. 2020, Article ID 5196302, 17 pages, 2020.

[29] Q. Q. Zeng, J. W. Cai, and Y. Xu, "Network pharmacologybased analysis of effective components and mechanism of Rhizoma coptidis in treating diabetes," Asian Pacific Journal of Tropical Biomedicine, vol. 11, no. 1, pp. 29-39, 2021.

[30] A. Nandy, S. Kar, and K. Roy, "Development of classificationand regression-based QSAR models andin silicoscreening of skin sensitisation potential of diverse organic chemicals," Molecular Simulation, vol. 40, no. 4, pp. 261-274, 2014.

[31] X. Guo, J. Ji, Z. Feng, X. Hou, Y. Luo, and Z. Mei, “A network pharmacology approach to explore the potential targets underlying the effect of sinomenine on rheumatoid arthritis," International Immunopharmacology, vol. 80, Article ID 106201, 2020.

[32] W. B. Dai, W. W. Peng, and X. W. Zhong, "Study on the mechanism of atractylodes lancea in the treatment of diabetes based on network pharmacology," Medicinal Plants, vol. 11, no. 4, pp. 33-37, 2020.

[33] Y. Wang, S. Wang, and J. H. He, "Transcriptomic analysis reveals essential microRNAs after peripheral nerve injury," Neural regeneration research, vol. 16, no. 9, pp. 1865-1870, 2021.

[34] S. Wu, D. Wu, Y. Pan, H. Liu, Z. Shao, and M. Wang, "Correlation between EZH2 and CEP55 and lung adenocarcinoma prognosis," Pathology, Research \& Practice, vol. 215, no. 2, pp. 292-301, 2019.

[35] J. Zhang, Y. Liu, and G. Shi, "Gene microarray analysis of expression profiles in Suberoyllanilide hyroxamic acid-treated Dendritic cells," Biochemical and Biophysical Research Communications, vol. 508, no. 2, pp. 392-397, 2019.

[36] F. F. Fan, F. F. Guo, and W. Zhang, "Molecular mechanism of Tibetan medicine Rhodiola crenulata prevents oxidative stress through network pharmacology," Zhongguo Zhongyao Zazhi, vol. 43, no. 09, pp. 1789-1797, 2018, in Chinese.

[37] G. Yu, L.-G. Wang, Y. Han, and Q.-Y. He, "clusterProfiler: an $\mathrm{R}$ Package for comparing biological themes among gene clusters," OMICS: A Journal of Integrative Biology, vol. 16, no. 5, pp. 284-287, 2012.

[38] J. Dong, L. Zhang, Y. Liu et al., "Luteolin decreases the pathogenicity of Aeromonas hydrophila via inhibiting the activity of aerolysin," Virulence, vol. 12, no. 1, pp. 165-176, 2021.

[39] X.-M. Zhang, H.-J. Zhang, M. Liu et al., "Cloning and immunosuppressive properties of an acyl-activating enzyme from the venom apparatus of tetrastichus brontispae (hymenoptera: eulophidae)," Toxins, vol. 11, no. 11, pp. 672-685, 2019.

[40] J. Li, X. B. Ma, and J. Shen, "Screening of active components from Chinese materia medica against SARS-CoV-2 based on literature mining and molecular docking," Chinese Traditional and Herbal Drugs, vol. 51, no. 4, pp. 845-850, 2020, in Chinese.

[41] F. H. Liu, S. J. Chen, and W. J. Ni, "Study on the computer virtual screening of antithrombotic active ingredients in 
Chuanxiong Rhizoma," Journal of Chinese Pharmaceutical Sciences, vol. 28, pp. 2182-2186, 2017, in Chinese.

[42] I. Radziejewska, M. Borzym-Kluczyk, and K. Leszczyńska, "Luteolin alters MUC1 extracellular domain, sT antigen, ADAM-17, IL-8, IL-10 and NF- $\kappa$ B expression in Helicobacter pylori-infected gastric cancer CRL-1739 cells: a preliminary study," Biomed Rep, vol. 14, no. 2, pp. 19-30, 2021.

[43] H. Zhang, X. Zhong, X. Zhang, D. Shang, Y. Zhou, and C. Zhang, "Enhanced anticancer effect of ABT-737 in combination with naringenin on gastric cancer cells," Experimental and Therapeutic Medicine, vol. 11, no. 2, pp. 669-673, 2016.

[44] J. S. Lv, Shi Jimo DuiYao. Beijing, People's Military Medical Press, Beijing, p. 34, 2005, in Chinese.

[45] P. A. Correa, "Human model of gastric carcinogenesisCancer," Res: Anthropology and Aesthetics, vol. 48, pp. 3554-3560, 1988.

[46] M. J. Hu and Z. F. Wang, "Treatment of Helicobacter pyloriassociated gastritis using traditional Chinese medicine," Infection International, vol. 7, no. 1, pp. 1-5, 2018.

[47] W. F. Wang, F. L. Liu, and X. T. Xia, "Effects of Zhizhu Pills on expression of PI3K and AKT in colon of mice with slow transmission and constipation of spleen deficiency syndrome," Journal of Traditional Chinese Medical Pharmacy, vol. 35, no. 6, pp. 2824-2828, 2020, in Chinese.

[48] Y. Liu and X. J. Li, "Experience of LI xuejun's in regulating gastrointestinal diseases with atractylodes macrocephala," Clinical Journal of Traditional Chinese Medicine, vol. 32, no. 05, pp. 866-869, 2020, in Chinese.

[49] S.-Q. Wang, C. Wang, L.-M. Chang et al., "Geridonin and paclitaxel act synergistically to inhibit the proliferation of gastric cancer cells through ROS-mediated regulation of the PTEN/PI3K/Akt pathway," Oncotarget, vol. 7, no. 45, pp. 72990-73002, 2016.

[50] Y. Zhang, J. B. Xiong, and H. P. Chen, "Research progresses of PTEN/PI3K/AKT signaling pathway in gastric cancer," Chongqing Med J, vol. 47, no. 19, pp. 2601-2603, 2018, in Chinese.

[51] D. Zhou, W. Liu, S. Liang et al., "Apoptin-derived peptide reverses cisplatin resistance in gastric cancer through the PI3K-AKT signaling pathway," Cancer Medicine, vol. 7, no. 4, pp. 1369-1383, 2018.

[52] I. Riquelme, O. Tapia, J. A. Espinoza et al., "The gene expression status of the PI3K/AKT/mTOR pathway in gastric cancer tissues and cell lines," Pathology and Oncology Research, vol. 22, no. 4, pp. 797-805, 2016.

[53] L. Liu, Y. Ye, and X. Zhu, "MMP-9 secreted by tumor associated macrophages promoted gastric cancer metastasis through a PI3K/AKT/Snail pathway," Biomedicine \& Pharmacotherapy, vol. 117, Article ID 109096, 2019.

[54] K. Taniguchi and M. Karin, "IL-6 and related cytokines as the critical lynchpins between inflammation and cancer," Seminars in Immunology, vol. 26, no. 1, pp. 54-74, 2014.

[55] F. Ma, M. D. Zhang, and Y. J. Wang, "Correlation between IL6 expression and epithelial-mesenchy-maltransitions in gastric cancer and its clinical significance," Chinese Journal of Practical Surgery, vol. 34, no. 3, pp. 259-263, 2014, in Chinese.

[56] X.-B. Jia, Q. Zhang, L. Xu, W.-J. Yao, and L. Wei, "Effect of malus asiatica nakai leaf flavonoids on the prevention of esophageal cancer in C57BL/6J mice by regulating the IL-17 signaling pathway," OncoTargets and Therapy, vol. 13, no. 13, pp. 6987-6996, 2020.
[57] M. Nie, Y. Wang, Z. Yu et al., "AURKB promotes gastric cancer progression via activation of CCND1 expression," Aging, vol. 12, no. 2, pp. 1304-1321, 2020.

[58] C. Song, Y. Han, H. Luo et al., "HOXA10 induces BCL2 expression, inhibits apoptosis, and promotes cell proliferation in gastric cancer," Cancer Medicine, vol. 8, no. 12, pp. 5651-5661, 2019.

[59] Y. Pu, T. Zhang, J. Wang et al., "Luteolin exerts an anticancer effect on gastric cancer cells through multiple signaling pathways and regulating miRNAs," Journal of Cancer, vol. 9, no. 20, pp. 3669-3675, 2018.

[60] F. Shi, D. Zhou, Z. Ji, Z. Xu, and H. Yang, "Anti-arthritic activity of luteolin in Freund's complete adjuvant-induced arthritis in rats by suppressing P2X4 pathway," ChemicoBiological Interactions, vol. 226, no. 226, pp. 82-87, 2015

[61] L. Bao, F. Liu, H.-b. Guo et al., "Naringenin inhibits proliferation, migration, and invasion as well as induces apoptosis of gastric cancer SGC7901 cell line by downregulation of AKT pathway," Tumor Biology, vol. 37, no. 8, pp. 11365-11374, 2016.

[62] Y. L. Chen and C. X. Wu, "Experimental study of Naringenin in promoting apoptosis of human gastric cancer SGC-7901 through PI3K/AKT signaling pathway," Chinese Journal of Coal Industry Medicine, vol. 23, no. 5, pp. 449-456, 2020, in Chinese. 